\title{
CXCR7 expression is associated with disease-free and disease-specific survival in cervical cancer patients
}

\author{
M Schrevel', R Karim ${ }^{2,3}$, NT ter Haar', SH van der Burg ${ }^{3}$, JBMZ Trimbos', GJ Fleuren', A Gorter, \\ and ES Jordanova'
}

'Department of Pathology, Leiden University Medical Center (LUMC), PO Box 9600, 2300 RC Leiden, The Netherlands; '2Department of Immunohaematology and Blood Transfusion/Center for Human and Clinical Genetics, Leiden University Medical Center (LUMC), PO Box 9600,2300 RC Leiden, The Netherlands; ${ }^{3}$ Department of Clinical Oncology, Leiden University Medical Center (LUMC), PO Box 9600, 2300 RC Leiden, The Netherlands;

${ }^{4}$ Department of Gynaecology, Leiden University Medical Center (LUMC), PO Box 9600, 2300 RC Leiden, The Netherlands

BACKGROUND: The CXC chemokine receptor (CXCR)7 is involved in tumour development and metastases formation. The aim of the present study was to determine protein expression of CXCR7, its putative co-receptors epidermal growth factor receptor (EGFR) and CXCR4, its predominant ligand CXCLI2, their co-dependency and their association with survival in cervical cancer patients. METHODS: CXC chemokine receptor 7, EGFR, CXCR4 and CXCLI2 expression were determined immunohistochemically in 103 paraffin-embedded, cervical cancers. Subsequently, associations with patient characteristics were assessed and survival analyses were performed.

RESULTS: CXC chemokine receptor 7 was expressed by $43 \%$ of tumour specimens, in a large majority of cases together with either EGFR or CXCR4 (double positive), or both (triple positive). The CXCR7 expression was associated with tumour size $(P=0.013)$, lymph node metastasis $(P=0.00 \mathrm{I})$ and EGFR expression $(P=0.009)$. CXC chemokine receptor 7 was independently associated with disease-free survival (hazard ratio $(\mathrm{HR})=4.3,95 \%$ confidence intervals $(\mathrm{Cl})$ I.7-I I.0, $P=0.002$ ), and strongly associated with disease-specific survival ( $\mathrm{HR}=3.9,95 \% \mathrm{Cl} 1.5-10.2, P=0.005)$.

CONCLUSION: CXC chemokine receptor 7 expression predicts poor disease-free and disease-specific survival in cervical cancer patients, and might be a promising new therapeutic marker. In a large majority of cases, CXCR7 is co-expressed with CXCR4 and/or EGFR, supporting the hypothesis that these receptors assist in CXCR7 signal transduction.

British Journal of Cancer (2012) 106, I520-1525. doi: I0.1038/bjc.20 I2.I I0 www.bjcancer.com

(C) 2012 Cancer Research UK

Keywords: cervical cancer; CXCLI2; CXCR4; CXCR7; EGFR; survival

Cervical cancer is the third most common type of cancer among women worldwide, accounting for $9 \%$ of all new cancer cases among females in 2008 (Jemal et al, 2011). Although screening for premalignant stages of cervical cancer and vaccination with the available human papillomavirus (HPV)-vaccines are good options to prevent cervical carcinogenesis, treatment options are limited when women present with advanced cancer stages. Partly, this is because the mechanisms involved in tumour cell invasion and metastasis formation, which strongly predict mortality rates, are not fully understood. Furthermore, markers that accurately predict response to therapy are limited. Additional predictive molecular markers for lymph node metastases and disease-free survival may help to elucidate these mechanisms.

A promising new marker is the CXC chemokine receptor (CXCR) 7. CXC chemokine receptor 7 is transcribed from the RDC1-gene on chromosome 2, where the genes encoding CXCR1, CXCR2 and CXCR4 are also localised (Balabanian et al, 2005). CXC chemokine receptor 7 is strongly expressed in many different tumour types and on tumour-associated vasculature, whereas expression in most normal tissues is weak or absent. The CXCR7 is

*Correspondence: Dr A Gorter; E-mail: A.Gorter@lumc.nl Received 25 January 2012; revised 6 March 2012; accepted 8 March 2012 involved in cell survival, cell adhesion, tumour development and metastases formation (Burns et al, 2006; Miao et al, 2007). Expression of CXCR7 has not been determined in cervical cancer, but has been associated with a higher tumour grade and more aggressive tumour growth in other cancer types, such as prostate cancer, non-small cell lung cancer, breast cancer, glioma and hepatocellular carcinoma (Miao et al, 2007; Wang et al, 2008; Iwakiri et al, 2009; Hattermann et al, 2010; Zheng et al, 2010). However, most studies have been performed on tumour cell lines and mouse models, and studies addressing the association between CXCR7 expression and prognosis in patient-derived tumour material are limited.

Two ligands bind to CXCR7, namely CXCL11 (interferoninducible T-cell $\alpha$ chemoattractant) and CXCL12 (stromal cellderived factor-1). In cell lines, activation of CXCR7 by CXCL12 induced trans-endothelial migration, whereas CXCR7 antagonists and CXCL11 both inhibited cellular migration (Zabel et al, 2011). Furthermore, CXCL12 expression was associated with overall and disease-free survival in pancreas carcinoma, suggesting that CXCL12 is the predominant ligand associated with CXCR7-mediated metastasis formation (Liang et al, 2010).

Conflicting results have been published on the intracellular signalling pathways of CXCR7, as ligand binding does not result in typical CXC receptor Gi-mediated signalling (Thelen and Thelen, 2008). It is still unclear whether the receptor is able to activate 
other signal transduction pathways. It has been proposed that CXCR7 heterodimerisation with other signalling receptors such as the epidermal growth factor receptor (EGFR) or CXCR4 is required to induce intracellular signalling (Hartmann et al, 2008; Décaillot et al, 2011; Singh and Lokeshwar, 2011). Both EGFR and CXCR4 expression have been shown to be associated with lymph node metastases and disease-free survival in cervical cancer (Kersemaekers et al, 1999; Kodama et al, 2007; Schrevel et al, 2011).

The aim of the present study was to determine protein expression of CXCR7, its putative co-receptors EGFR and CXCR4, its main ligand CXCL12, their co-dependency and their association with survival in cervical cancer patients. Therefore, we immunohistochemically determined CXCR7, EGFR, CXCR4 and CXCL12 expression, and assessed the association between protein expression and patient characteristics. Furthermore, we analysed whether (co-)expression of CXCR7, EGFR and CXCR4 was associated with disease-specific and disease-free survival.

\section{MATERIALS AND METHODS}

\section{Subjects}

Formalin-fixed, paraffin-embedded primary tumour tissue samples from 103 cervical cancer patients who underwent radical hysterectomy with lymphadenectomy between January 1985 and December 1999 were collected from the archives of the Department of Pathology, Leiden University Medical Center (Leiden, the Netherlands). Patients had not received radiotherapy or chemotherapy before surgery. Postoperative radiotherapy was indicated in patients with lymph node metastasis, parametrial involvement or positive resection margins. Clinical and follow-up data were taken from patient medical records. Disease-specific survival and disease-free survival were assessed to determine the time to cancer-related death and disease recurrence, respectively. Disease-specific survival time was assessed from the date of surgery to the date of cancer-related death or the date of the last follow-up visit for censored observations. For disease-specific survival, patients who died of a cause unrelated to cervical cancer were considered as censored observations at the date of death. The end point for disease-free survival was the date of local or regional recurrence or the date of distant metastasis.

Tumours were HPV-typed by general primer PCR and sequencing, as described by Koopman et al (1999). In addition to the tumour samples, two specimens of normal cervical epithelium were obtained from two patients with no history of cervical cancer. This number of healthy tissue samples was sufficient, as the staining of CXCR4, CXCR7 and CXCL12 was expected to be similar for all normal cervical epithelium specimens. Tissue samples were used according to the guidelines of the Ethical Committee of the Leiden University Medical Center.

\section{Immunohistochemistry}

For immunohistochemical analysis, $4 \mu \mathrm{m}$ tissue microarray (TMA) slides consisting of triplicate punches of 103 cervical cancer patients were deparaffinised and rehydrated. Endogenous peroxidase was blocked with $0.3 \%$ hydrogen peroxide $\left(\mathrm{H}_{2} \mathrm{O}_{2}\right)$ for $20 \mathrm{~min}$. Antigen retrieval was performed in $0.01 \mathrm{~m}$ citrate buffer $(\mathrm{pH}=6.0$, $12 \mathrm{~min}$, microwave oven). Subsequently, slides were incubated overnight at room temperature with anti-CXCR4 $(1: 600$, IgG2a, clone 2F1, Abnova, Heidelberg, Germany), anti-CXCR7 (1:50, IgG1, clone 11G8, R\&D Systems, Abingdon, UK) or anti-CXCL12 (1:50, IgG1, clone 79018, R\&D Systems), diluted in phosphatebuffered saline (PBS) containing $1 \%$ bovine serum albumin. After washing with PBS, the TMA slides were incubated for $30 \mathrm{~min}$ with BrightVision-Poly/HRP (Immunologic, Duiven, the Netherlands). Immunoreactions were visualised using $0.5 \%$
3, $3^{\prime}$-diamino-benzidine-tetra-hydrochloride and $0.002 \% \mathrm{H}_{2} \mathrm{O}_{2}$ in Tris- $\mathrm{HCl}$, after which the slides were counterstained with haematoxylin. Immunoreactivity was scored as negative, weak, moderate or strong staining intensity. Immunohistochemistry for EGFR was performed as previously described, and EGFR was scored for membrane staining intensity (Schrevel et al, 2011).

\section{Statistical analyses}

Statistical analyses were performed using the SPSS program (Version 17.0 for Windows; SPSS Inc., Chicago, IL, USA). Significance tests were two-sided and statistical significance was assumed when $P<0.05$, corresponding to $95 \%$ confidence intervals (CI). To assess whether CXCR7 expression was associated with expression of its ligand CXCL12 or its proposed co-receptors EGFR and CXCR4, the Spearman's rank correlation test was performed, as the intensity scores for CXCR7, EGFR, CXCR4 and CXCL12 were ordinal. The $\chi^{2}$ test was used to determine whether CXCR7, EGFR, CXCR4 and CXCL12 expression was associated with clinicopathological characteristics, with protein expression data recoded into binary variables. Univariate Cox-regression analysis was performed to assess the association between clinicopathological parameters, CXCR7, EGFR, CXCR4 and CXCL12 expression, and disease-free survival. All analyses were repeated for diseasespecific survival. Multivariate Cox-regression analysis was performed to determine whether CXCR7 expression was independently associated with disease-free survival, when correcting for HPV-type, histopathological diagnosis, tumour size, infiltration depth, parametrial invasion, vasoinvasion, lymph node metastasis, resection margins and postoperative radiotherapy. Corresponding survival curves were estimated by the Kaplan-Meier method.

\section{RESULTS}

\section{Patient characteristics in relation to CXCR7, EGFR, CXCR4 and CXCL12 expression in cervical cancer}

Immunohistochemical staining of CXCR7, CXCR4 and CXCL12 was observed to be both cytoplasmic and membranous in cervical cancer specimens. Normal cervical epithelium stained weakly for CXCR7, CXCR4 and CXCL12, with strong CXCL12 expression in cells of the basal layer. Representative examples of positive and negative CXCR7, EGFR, CXCR4 and CXCL12 cervical cancer specimens are shown in Figure 1. The distribution of staining intensities is shown in Table 1. CXC chemokine receptor 7, EGFR and CXCL12 had a wider range in staining intensity, when compared with CXCR4, as no strong positives were observed for CXCR4. To assess whether CXCR7 expression was associated with expression of its ligand CXCL12 or its proposed co-receptors EGFR and CXCR4, the Spearman's rank correlation coefficient ( $\rho$; rho) was determined. CXC chemokine receptor 7 expression was significantly correlated with EGFR expression $(\rho=0.272$, $P=0.009)$, but not with CXCR4 expression $(\rho=0.117, P=0.259)$ or CXCL12 expression $(\rho=-0.028, P=0.790)$. Compared with an ideal correlation of 1 , the observed Spearman's $\rho$ of 0.272 indicates a moderate correlation.

Clinicopathological characteristics of the 103 cervical cancer patients are summarised in Table 2. The median age at the time of diagnosis was 48 years (range, 24-87). Positive CXCR7 expression was associated with tumour size $\geqslant 40 \mathrm{~mm}$ (odds ratio $(\mathrm{OR})=2.9$, $95 \%$ confidence interval $(\mathrm{CI}) 1.2-6.8, P=0.013)$ and lymph node positivity at the time of surgery $(\mathrm{OR}=5.7,95 \% \mathrm{CI} 2.0-16.1$, $P=0.001)$. CXC chemokine receptor 7 expression was observed more frequently in squamous carcinoma than in adeno-/adenosquamous carcinoma $(\mathrm{OR}=2.6,95 \% \mathrm{CI} 1.1-6.0, P=0.025)$. Both EGFR and CXCR4 expression were observed more frequently in squamous/adenosquamous carcinoma than in adenocarcinoma 

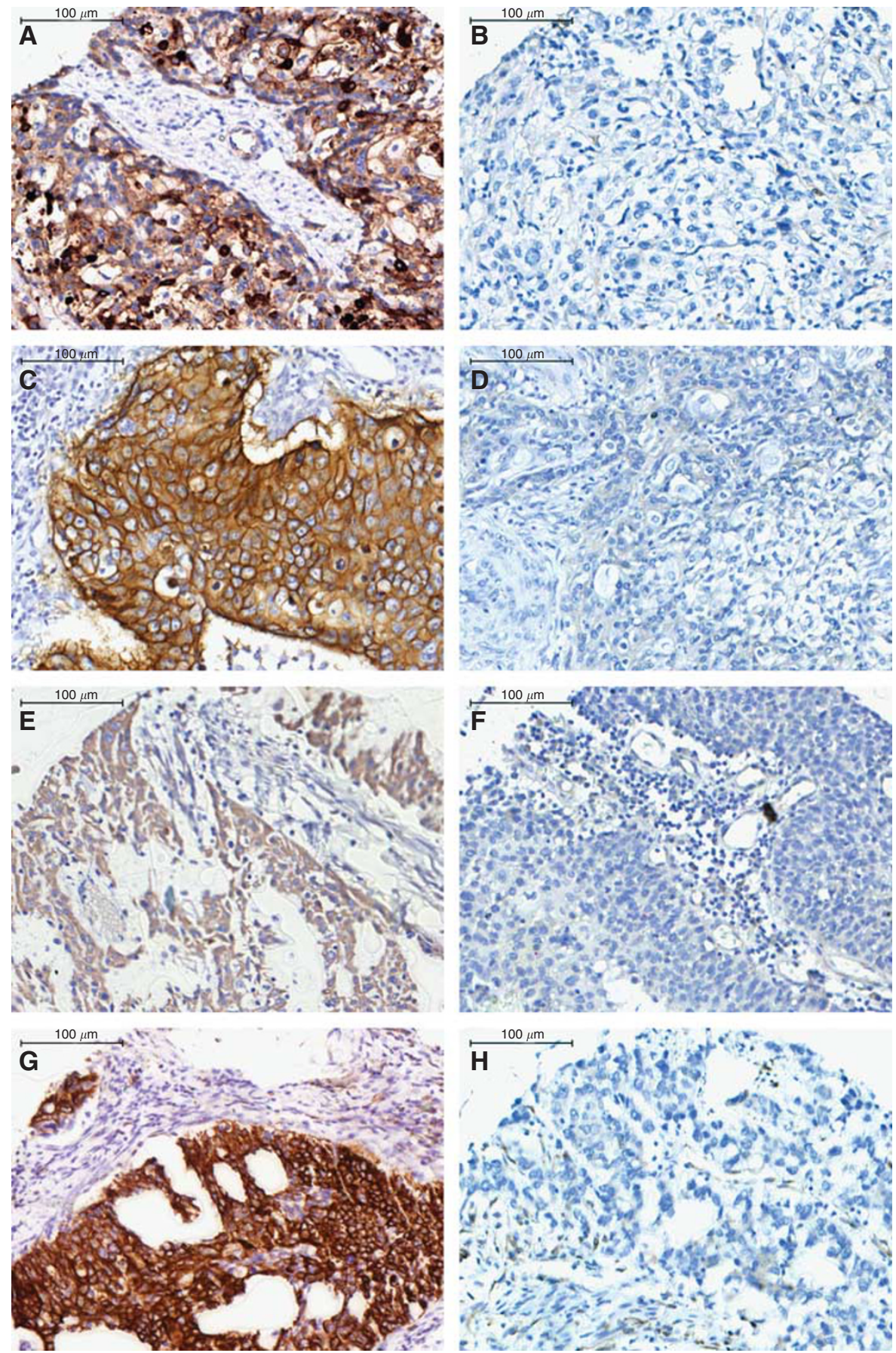

Figure I Representative examples of positive $(\mathbf{A})$ and negative $(\mathbf{B})$ CXCR7 staining, positive $(\mathbf{C})$ and negative (D) EGFR staining, positive (E) and negative $(\mathbf{F})$ CXCR4 staining, and positive $(\mathbf{G})$ and negative $(\mathbf{H})$ CXCLI 2 staining in the epithelial compartment of squamous cell carcinoma of the cervix.

(OR 26.6, 95\% CI 3.3-217.4, $P<0.001$ and $\mathrm{OR}=6.4,95 \% \mathrm{CI}$ 1.6-25.7, $P=0.008$, respectively). No other associations were observed between CXCR7, CXCR4, CXCL12 and clinicopathological characteristics. As previously described, EGFR expression was associated with tumour size, parametrial invasion, vasoinvasion and lymph node metastasis (Schrevel et al, 2011).

\section{Disease-specific and disease-free survival for CXCR7, EGFR, CXCR4 and CXCL12}

Median follow-up time was 137 months (range 5-266) for all patients and 156 months (range 8-266) for patients alive at the time of data collection. Of the 24 patients with disease recurrence, a combination of local recurrences $(n=4)$, regional recurrences $(n=3)$ and distant metastases $(n=20)$ was observed. Of the 34 patients who died during the follow-up period, 22 deaths could be attributed to cervical cancer. Five-year disease-free and disease-specific survival rates for the whole group were $77 \% \quad($ s.e. $=4)$ and $79 \% \quad($ s.e. $=4)$, respectively. Univariate Cox-regression analysis for all clinicopathological characteristics and disease-free survival is shown in Table 2. Multivariate regression analysis for all clinicopathological characteristics showed that only tumour size (hazard ratio $(\mathrm{HR})=4.8,95 \%$ CI 1.8-12.3, $P=0.001)$ and lymph node metastasis $(\mathrm{HR}=5.4,95 \%$ 
Table I CXCR7, EGFR, CXCR4 and CXCLI2 expression in cervical cancer patients

\begin{tabular}{lc}
\hline Expression & $\mathbf{N}(\%)^{\mathbf{a}}$ \\
\hline CXCR7 & \\
Negative & $58(57)$ \\
Weak & $18(18)$ \\
Moderate & $20(20)$ \\
Strong & $5(5)$ \\
EGFR & \\
Negative & $19(20)$ \\
Weak & $16(17)$ \\
Moderate & $31(33)$ \\
Strong & $28(30)$ \\
CXCR4 & \\
Negative & $36(37)$ \\
Weak & $51(53)$ \\
Moderate & $10(10)$ \\
Strong & $0(0)$ \\
CXCLI2 & \\
Negative & $13(14)$ \\
Weak & $35(38)$ \\
Moderate & $28(31)$ \\
Strong & $16(17)$ \\
\hline
\end{tabular}

Abbreviations: $\mathrm{CXCR}=\mathrm{CXC}$ chemokine receptor; $\mathrm{EGFR}=$ epidermal growth factor receptor. ${ }^{\text {TT}}$ Total number of assessed cases is 101 for CXCR7, 94 for EGFR, 97 for CXCR4 and 92 for CXCL 12. Protein expression was determined through analysis of an immunohistochemically stained tissue array, as described in the Materials and Methods section. Immunoreactivity was scored as negative, weak, moderate or strong staining intensity.

CI $2.3-12.7, \quad P<0.001)$ were independently associated with disease-free survival.

Univariate Cox-regression analysis for CXCR7 showed a strong association between positive CXCR7 expression and disease recurrence, with $63 \%$ vs $90 \%$ disease-free survival for CXCR7positive and CXCR7-negative cases, respectively, ( $\mathrm{HR}=4.3,95 \%$ CI 1.7-11.0, $P=0.002$, Figure 2A, Table 3). CXC chemokine receptor 7 expression was also associated with disease-specific survival $(\mathrm{HR}=3.9,95 \%$ CI $1.5-10.2, P=0.005)$. CXC chemokine receptor 4 and CXCL12 expression were not associated with disease-free (Table 3) nor disease-specific survival (data not shown). There was a trend for a positive association between EGFR expression and disease-free survival $(\mathrm{HR}=2.3,95 \% \mathrm{CI} 0.9-6.4$, $P=0.095$, Table 3).

Multivariate regression analysis showed that only CXCR7 expression $(\mathrm{HR}=3.4,95 \% \mathrm{CI} 1.1-10.5, P=0.030)$, tumour size per $\mathrm{mm}$ increase in tumour size $(\mathrm{HR}=1.1,95 \%$ CI $1.0-1.1$, $P=0.001)$ and lymph node metastasis at the time of surgery ( $\mathrm{HR}=4.5,95 \%$ CI $1.2-16.8, P=0.024)$ were independent predictors of disease-free survival, when correcting for HPV-type, histopathological diagnosis, tumour size, infiltration depth, parametrial invasion, vasoinvasion, lymph node metastasis, resection margins and postoperative radiotherapy. Multivariate regression analysis for CXCR7, EGFR, CXCR4 and CXCL12 expression showed that only CXCR7 was independently associated with disease-recurrence $(\mathrm{HR}=4.2,95 \% \mathrm{CI} 1.5-12.0, P=0.007)$.

\section{Disease-free survival for co-expression of CXCR7, EGFR, CXCR4 and CXCL12}

To assess whether co-expression of CXCR7 and its ligand CXCL12 was associated with disease-free or disease-specific survival, groups were made for CXCR7-negative cases $(n=58)$ and CXCR7-positive cases with $(n=33)$ or without CXCL12 expression
Table 2 Disease-free survival for clinicopathological parameters

\begin{tabular}{|c|c|c|c|c|c|}
\hline Variables & $\mathbf{N}^{\mathbf{a}}$ & DFS (\%) & HR & $95 \% \mathrm{Cl}$ & $P$-value \\
\hline \multicolumn{6}{|l|}{ HPV-type } \\
\hline Negative & 9 & 89 & - & - & - \\
\hline 16 & 54 & 76 & 2.2 & $0.3-16.3$ & 0.465 \\
\hline 18 & 23 & 65 & 3.7 & $0.5-29.7$ & 0.216 \\
\hline Other & 17 & 88 & 1.1 & $0.1-12.0$ & 0.944 \\
\hline \multicolumn{6}{|c|}{ Histopathology } \\
\hline SCC & 62 & 74 & 0.7 & $0.3-1.7$ & 0.447 \\
\hline$A(S)$ & 41 & 81 & & & \\
\hline \multicolumn{6}{|l|}{ Tumour size } \\
\hline$<40 \mathrm{~mm}$ & 59 & 90 & 6.1 & $2.4-15.6$ & $<0.001$ \\
\hline$\geqslant 40 \mathrm{~mm}$ & 38 & 55 & & & \\
\hline \multicolumn{6}{|c|}{ Infiltration depth } \\
\hline$<15 \mathrm{~mm}$ & 56 & 88 & 3.7 & $1.5-9.0$ & 0.003 \\
\hline$\geqslant 15 \mathrm{~mm}$ & 46 & 63 & & & \\
\hline \multicolumn{6}{|c|}{ Parametrial invasion } \\
\hline Negative & 94 & 81 & 5.2 & $2.0-13.1$ & 0.001 \\
\hline Positive & 9 & 33 & & & \\
\hline \multicolumn{6}{|l|}{ Vasoinvasion } \\
\hline Negative & 48 & 83 & 2.1 & $0.9-4.9$ & 0.086 \\
\hline Positive & 52 & 69 & & & \\
\hline \multicolumn{6}{|c|}{ Lymph node metastasis } \\
\hline Negative & 79 & 87 & 7.2 & $3.2-16.3$ & $<0.001$ \\
\hline Positive & 24 & 42 & & & \\
\hline \multicolumn{6}{|c|}{ Resections margins } \\
\hline Negative & 77 & 83 & 3.0 & $1.4-6.8$ & 0.007 \\
\hline Positive & 26 & 58 & & & \\
\hline \multicolumn{6}{|c|}{ Postoperative radiotherapy } \\
\hline No & 47 & 92 & 5.1 & $1.7-15.0$ & 0.003 \\
\hline Yes & 56 & 64 & & & \\
\hline
\end{tabular}

Abbreviations: $A(S)=$ adeno(squamous) carcinoma; $D F S=$ disease-free survival; $\mathrm{HR}=$ hazard ratio; $95 \% \mathrm{Cl}=95 \%$ confidence interval; $\mathrm{HPV}=$ human papillomavirus; $\mathrm{SCC}=$ squamous cell carcinoma. Univariate Cox-regression analysis for disease-free

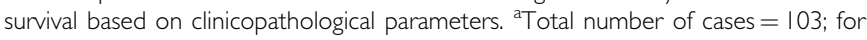
some variables, data were not available for all patients. The bold entries place emphasis on statistically significant $P$-values.

$(n=5)$. Survival analysis showed no difference between CXCR7positive/CXCL12-positive and CXCR7-positive/CXCL12-negative cases (data not shown). To investigate whether co-expression of CXCR7 and EGFR, CXCR4 or both was associated with disease-free or disease-specific survival, groups were made for CXCR7-negative cases $(n=58)$, CXCR7-positive cases with no EGFR or CXCR4 expression (single positive, $n=4$ ), CXCR7-positive cases with either EGFR or CXCR4 expression (double positive, $n=16$ ) and CXCR7-positive cases with both EGFR and CXCR4 expression (triple positive, $n=21$ ). As the survival curves for the 4 CXCR7 single-positive cases were comparable to the 58 CXCR7-negative cases, these groups were combined for further analyses. Univariate Cox-regression analysis showed equally increased risks of disease recurrence for both double- and triple-positive cases when compared with CXCR7-negative/single-positive cases $(\mathrm{HR}=3.6$, 95\% CI $1.2-10.7, P=0.022$, and $\mathrm{HR}=4.2,95 \%$ CI 1.5-11.7, $P=0.006$, respectively, Figure $2 \mathrm{~B}$, Table 3$)$. As expression of CXCR7 was observed more frequently in squamous cell carcinoma than in other histopathological types, regression analysis was also performed for this subgroup. In squamous cell carcinomas, the risk between disease-free survival and CXCR7-negative/singlepositive cases, double-positive cases and triple-positive cases increased in an additive fashion (double: $\mathrm{HR}=2.7,95 \% \mathrm{CI}$ $0.6-12.1, P=0.193$, triple: $\mathrm{HR}=5.8,95 \%$ CI $1.5-22.4, P=0.011$, Figure 2C, Table 3). Survival analysis for disease-specific survival showed similar results (data not shown). 

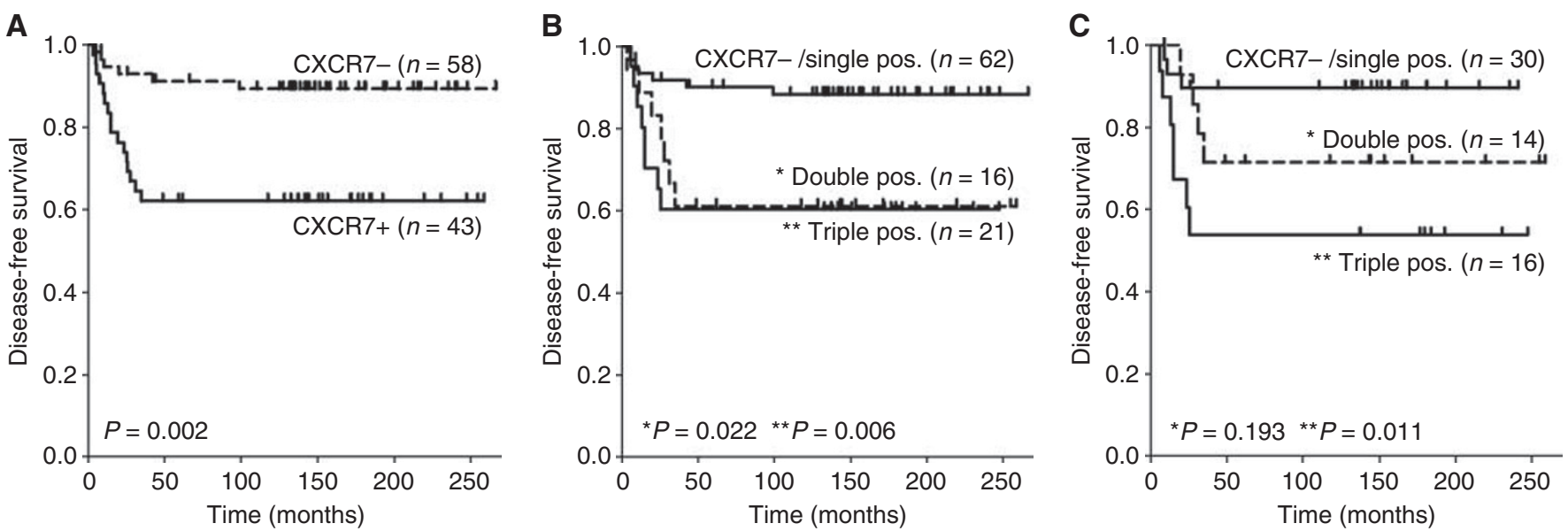

Figure 2 Disease-free survival in cervical cancer patients with positive or negative CXCR7 expression (A), CXCR7-negative/CXCR7 single-positive cases, CXCR7-positive, and either EGFR- or CXCR4-positive (double positive) cases and CXCR7-, EGFR- and CXCR4-positive (triple positive) cases in all patients (B) and in patients with squamous cell carcinoma (C). P-values were obtained using Cox-regression analysis; see Table 3 for HRs and Cls.

Table 3 Disease-free survival for CXCR7, EGFR, CXCR4 and CXCLI2 protein expression

\begin{tabular}{|c|c|c|c|c|c|}
\hline Variables & $N^{a}$ & DFS (\%) & HR & $95 \% \mathrm{Cl}$ & $P$-value \\
\hline \multicolumn{6}{|l|}{ CXCR7 } \\
\hline Negative & 58 & 90 & 4.3 & $1.7-11.0$ & 0.002 \\
\hline Positive & 43 & 63 & & & \\
\hline \multicolumn{6}{|l|}{ EGFR } \\
\hline Low & 35 & 86 & 2.3 & $0.9-6.4$ & 0.095 \\
\hline High & 59 & 71 & & & \\
\hline \multicolumn{6}{|l|}{ CXCR4 } \\
\hline Negative & 36 & 78 & 1.2 & $0.5-2.9$ & 0.625 \\
\hline Positive & 61 & 74 & & & \\
\hline \multicolumn{6}{|l|}{ CXCLI2 } \\
\hline Negative & 13 & 69 & 0.8 & $0.3-2.3$ & 0.653 \\
\hline Positive & 79 & 75 & & & \\
\hline \multicolumn{6}{|l|}{ CXCR7/EGFR/CXCR4 } \\
\hline CXCR7 negative/single positive & 62 & 89 & - & - & - \\
\hline Double positive & 16 & 63 & 3.6 & $1.2-10.7$ & 0.022 \\
\hline Triple positive & 21 & 62 & 4.2 & $1.5-11.7$ & 0.006 \\
\hline \multicolumn{6}{|l|}{ CXCR7/EGFR/CXCR4 in SCC } \\
\hline CXCR7 negative/single positive & 30 & 90 & - & - & - \\
\hline Double positive & 14 & 71 & 2.7 & $0.6-12.1$ & 0.193 \\
\hline Triple positive & 16 & 56 & 5.8 & $1.5-22.4$ & 0.011 \\
\hline
\end{tabular}

Abbreviations: $\mathrm{CXCR}=\mathrm{CXC}$ chemokine receptor, $\mathrm{DFS}=$ disease-free survival; $\mathrm{EGFR}=$ epidermal growth factor receptor, $\mathrm{HR}=$ hazard ratio; $95 \% \mathrm{Cl}=95 \%$ confidence interval; SCC $=$ squamous cell carcinoma. ${ }^{a}$ Total number of cases $=103$; for some variables, data were not available for all patients. Univariate Cox-regression analysis for disease-free survival based on the status of CXCR7, EGFR, CXCR4 and/or CXCLI2 protein expression. CXCR7, CXCR4 and CXCLI2 expression are divided into negative (intensity score of 0 ) and positive (intensity scores of I, 2 and 3) groups. EGFR expression was divided into low (intensity scores of 0 and I) and high (intensity scores of 2 and 3) expression groups. In addition, co-expression of CXCR7, EGFR and CXCR4 was analysed as follows: CXCR7-negative cases $(n=58)$ and CXCR7 single-positive cases $(n=4)$ were combined and compared with double-positive cases (i.e. CXCR7-positive cases with either high EGFR expression or positive CXCR4 expression), and triple positive cases (i.e. CXCR7-positive cases with both high EGFR expression and positive CXCR4 expression). This analysis was also performed after selection of patients with SCC. The bold entries place emphasis on statistically significant $P$-values

\section{DISCUSSION}

The present study showed for the first time that CXCR7 was independently associated with disease recurrence in cervical cancer. In addition, CXCR7 expression was associated with tumour size and lymph node status at the time of surgery. These findings suggest that CXCR7 expression leads to more aggressive tumour growth and metastasis formation.

CXC chemokine receptor 7 is frequently expressed on embryonic and neoplastic transformed cells, but undetectable or expressed at very low levels in normal adult tissues (Burns et al, 2006; Miao et al, 2007). Experiments using mouse models showed that increased CXCR7 expression resulted in a larger tumour size and an enhanced metastatic potential (Miao et al, 2007). Previously, CXCR7 has been shown to be associated with disease-specific and disease-free survival in non-small cell lung cancer (Iwakiri et al, 2009). In the present study, we showed that in cervical cancer as well, disease-free survival was lower in CXCR7-positive cases (63\%) than in CXCR7-negative cases $(90 \%, \mathrm{HR}=4.3,95 \% \mathrm{CI}$ $1.7-11.0, P=0.002)$, even when adjusting for other prognostic factors. Disease-specific survival was also strongly associated with CXCR7 expression. Therefore, CXCR7 might be used as a prognostic marker to predict disease recurrence, with additive predictive value to lymph node status and tumour size.

The current data also showed that CXCR7 was often coexpressed with EGFR or CXCR4 (double positive) or both (triple positive), with only four single-positive cases, supporting the hypothesis that CXCR7 requires other molecules for optimal intracellular signalling. Sierro et al (2007) have shown that CXCR7 forms heterodimers with CXCR4 and Singh and Lokeshwar (2011) have shown that CXCR7 is capable of forming heterodimers with EGFR. Activation of HEK293 cells co-expressing CXCR7 and CXCR4 results in a stronger calcium flux when compared with cells expressing CXCR4 alone, whereas CXCR7 was unable to induce calcium flux by itself (Burns et al, 2006; Sierro et al, 2007). However, CXCR7 is capable of signalling through MAPK/AKT pathways, indicating that EGFR, which also signals through MAPK/AKT, might be an alternative co-receptor for CXCR7 (Grymula et al, 2010). Interestingly, in CXCR7- and CXCR4positive rhabdomyosarcoma cells, CXCL12-induced chemotaxis could still be observed after blocking CXCR4, although strongly reduced when compared with controls (Grymula et al, 2010). Similarly, in CXCR7-positive, CXCR4-negative 4T1 breast cancer cells, CXCR7 expression promoted tumour growth and progression of lung metastases in mice (Miao et al, 2007). As rhabdomyosarcoma cells and 4T1 breast cancer cells are both EGFR-positive, CXCR7 might signal through heterodimerisation with EGFR in case CXCR4 is absent or blocked (Dykxhoorn et al, 2009; Herrmann et al, 2010). However, although our data support this hypothesis, as a positive correlation was observed between CXCR7 and EGFR expression, functional studies on cervical cancer 
cell lines are required to determine whether CXCR7 and EGFR are indeed co-dependent for signal transduction. Furthermore, although HRs were similar for double- and triple-positive cases in the whole study group, HRs increased in an additive fashion in squamous cell carcinomas, which indicates that molecular mechanisms regulating metastasis formation may differ between histopathological subtypes.

In conclusion, CXCR7 expression was strongly associated with tumour size, lymph node metastasis, disease recurrence and poor disease-specific survival, suggesting that CXCR7 expression leads to a biologically more aggressive tumour. CXC chemokine receptor 7 might be a promising new prognostic marker in cervical

\section{REFERENCES}

Balabanian K, Lagane B, Infantino S, Chow KY, Harriague J, Moepps B, Arenzana-Seisdedos F, Thelen M, Bachelerie F (2005) The chemokine SDF-1/CXCL12 binds to and signals through the orphan receptor RDC1 in T lymphocytes. J Biol Chem 280(42): 35760-35766

Burns JM, Summers BC, Wang Y, Melikian A, Berahovich R, Miao Z, Penfold ME, Sunshine MJ, Littman DR, Kuo CJ, Wei K, McMaster BE, Wright K, Howard MC, Schall TJ (2006) A novel chemokine receptor for SDF-1 and I-TAC involved in cell survival, cell adhesion, and tumor development. J Exp Med 203(9): 2201-2213

Décaillot FM, Kazmi MA, Lin Y, Ray-Saha S, Sakmar TP, Sachdev P (2011) CXCR7/CXCR4 heterodimer constitutively recruits beta-arrestin to enhance cell migration. J Biol Chem 286(37): 32188-32197

Dykxhoorn DM, Wu Y, Xie H, Yu F, Lal A, Petrocca F, Martinvalet D Song E, Lim B, Lieberman J (2009) miR-200 enhances mouse breast cancer cell colonization to form distant metastases. PLoS One 4(9): e7181

Grymula K, Tarnowski M, Wysoczynski M, Drukala J, Barr FG, Ratajczak J, Kucia M, Ratajczak MZ (2010) Overlapping and distinct role of CXCR7SDF-1/ITAC and CXCR4-SDF-1 axes in regulating metastatic behavior of human rhabdomyosarcomas. Int J Cancer 127(11): 2554-2568

Hartmann TN, Grabovsky V, Pasvolsky R, Shulman Z, Buss EC, Spiegel A, Nagler A, Lapidot T, Thelen M, Alon R (2008) A crosstalk between intracellular CXCR7 and CXCR4 involved in rapid CXCL12-triggered integrin activation but not in chemokine-triggered motility of human $\mathrm{T}$ lymphocytes and CD34 + cells. J Leukoc Biol 84(4): 1130-1140

Hattermann K, Held-Feindt J, Lucius R, Müerköster SS, Penfold ME Schall TJ, Mentlein R (2010) The chemokine receptor CXCR7 is highly expressed in human glioma cells and mediates antiapoptotic effects. Cancer Res 70(8): 3299-3308

Herrmann D, Seitz G, Warmann SW, Bonin M, Fuchs J, Armeanu-Ebinger S (2010) Cetuximab promotes immunotoxicity against rhabdomyosarcoma in vitro. I Immunother 33(3): 279-286

Iwakiri S, Mino N, Takahashi T, Sonobe M, Nagai S, Okubo K, Wada H, Date H, Miyahara R (2009) Higher expression of chemokine receptor CXCR7 is linked to early and metastatic recurrence in pathological stage I nonsmall cell lung cancer. Cancer 115(11): 2580-2593

Jemal A, Bray F, Center MM, Ferlay J, Ward E, Forman D (2011) Global cancer statistics. CA Cancer J Clin 61(2): 69-90

Kersemaekers AM, Fleuren GJ, Kenter GG, van den Broek LJ, Uljee SM, Hermans J, van de Vijver MJ (1999) Oncogene alterations in carcinomas of the uterine cervix: overexpression of the epidermal growth factor receptor is associated with poor prognosis. Clin Cancer Res 5(3): 577-586 cancer and may serve as a potential therapeutic target. CXC chemokine receptor 7 is often co-expressed with CXCR4 and/or EGFR, which supports the hypothesis that these receptors assist in CXCR7 signal transduction.

\section{ACKNOWLEDGEMENTS}

We gratefully acknowledge Jan Molenaar for his help with the collection of follow-up data, Michelle Osse for her technical support and Judith Boer for helpful discussions and supervision.

Kodama J, Hasengaowa, Kusumoto T, Seki N, Matsuo T, Ojima Y, Nakamura K, Hongo A, Hiramatsu Y (2007) Association of CXCR4 and CCR7 chemokine receptor expression and lymph node metastasis in human cervical cancer. Ann Oncol 18(1): 70-76

Koopman LA, Szuhai K, van Eendenburg JD, Bezrookove V, Kenter GG, Schuuring E, Tanke H, Fleuren GJ (1999) Recurrent integration of human papillomaviruses 16,45 , and 67 near translocation breakpoints in new cervical cancer cell lines. Cancer Res 59(21): 5615-5624

Liang JJ, Zhu S, Bruggeman R, Zaino RJ, Evans DB, Fleming JB, Gomez HF, Zander DS, Wang H (2010) High levels of expression of human stromal cell-derived factor-1 are associated with worse prognosis in patients with stage II pancreatic ductal adenocarcinoma. Cancer Epidemiol Biomarkers Prev 19(10): 2598-2604

Miao Z, Luker KE, Summers BC, Berahovich R, Bhojani MS, Rehemtulla A, Kleer CG, Essner JJ, Nasevicius A, Luker GD, Howard MC, Schall TJ (2007) CXCR7 (RDC1) promotes breast and lung tumor growth in vivo and is expressed on tumor-associated vasculature. Proc Natl Acad Sci USA 104(40): 15735-15740

Schrevel M, Gorter A, Kolkman-Uljee SM, Trimbos JB, Fleuren GJ, Jordanova ES (2011) Molecular mechanisms of epidermal growth factor receptor overexpression in patients with cervical cancer. Mod Pathol 24(5): 720-728

Sierro F, Biben C, Martinez-Muñoz L, Mellado M, Ransohoff RM, Li M, Woehl B, Leung H, Groom J, Batten M, Harvey RP, Martinez A Mackay CR, Mackay F (2007) Disrupted cardiac development but normal hematopoiesis in mice deficient in the second CXCL12/SDF-1 receptor, CXCR7. Proc Natl Acad Sci USA 104(37): 14759-14764

Singh RK, Lokeshwar BL (2011) The IL-8-regulated chemokine receptor CXCR7 stimulates EGFR signaling to promote prostate cancer growth. Cancer Res 71(9): 3268-3277

Thelen M, Thelen S (2008) CXCR7, CXCR4 and CXCL12: an eccentric trio? J Neuroimmunol 198(1-2): 9-13

Wang J, Shiozawa Y, Wang J, Wang Y, Jung Y, Pienta KJ, Mehra R Loberg R, Taichman RS (2008) The role of CXCR7/RDC1 as a chemokine receptor for CXCL12/SDF-1 in prostate cancer. J Biol Chem 283(7): 4283-4294

Zabel BA, Lewén S, Berahovich RD, Jaén JC, Schall TJ (2011) The novel chemokine receptor CXCR7 regulates trans-endothelial migration of cancer cells. Mol Cancer 10: 73

Zheng K, Li HY, Su XL, Wang XY, Tian T, Li F, Ren GS (2010) Chemokine receptor CXCR7 regulates the invasion, angiogenesis and tumor growth of human hepatocellular carcinoma cells. J Exp Clin Cancer Res 29: 31

This work is published under the standard license to publish agreement. After 12 months the work will become freely available and the license terms will switch to a Creative Commons Attribution-NonCommercial-Share Alike 3.0 Unported License. 